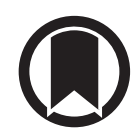

CrossMark

\section{Residual symptoms and lower lung function in patients recovering from SARS-CoV-2 infection}

To the Editor:

Initial reports of severe acute respiratory syndrome coronavirus 2 (SARS-CoV-2) infection indicate only mild disease in $81 \%$ of symptomatic cases [1]. Nearly half of the infected individuals do not seem to develop clinical symptoms at all, depending on the cohort investigated [2]. Comorbidities and older age were identified early during the pandemic as predictors for severe or critical disease [3], which is found in about $19 \%$ [1]. Independently from disease severity, little is known about longer-term outcomes as well as resolution of symptoms. We therefore set out to systematically evaluate residual symptoms and lung function impairment in patients after SARS-CoV-2 infection.

Patients were prospectively included for cross-sectional follow-up after PCR-confirmed SARS-CoV-2 infection. The protocol was approved by the ethics committee of the Medical Faculty Heidelberg (Heidelberg University) as part of an exploratory project and registered at clinicaltrials.gov (NCT04456075).

All subjects underwent spirometry and body plethysmography between March and June 2020 after written informed consent was obtained. Transfer factor of the lung for carbon monoxide $\left(T_{\mathrm{LCO}}\right)$ was determined using the single-breath technique. Clinical information was acquired at follow-up and retrospectively for the time of infection using a structured questionnaire. Global Lung Function Initiative (spirometry, $T_{\mathrm{LCO}}$ ) [4] and European Community for Steel and Coal (body plethysmography) [5] reference values were used. Unenhanced chest computed tomography (CT) was performed at follow-up when clinically indicated and within 1 week of lung function testing.

Mean values are given with standard deviation and between-group differences were assessed by $\mathrm{t}$-test. Fisher's exact test was used for categorical data. An alpha error of less than 5\% in two-sided testing was considered statistically significant. R Statistical Software (v3.6.1, Foundation for Statistical Computing, Vienna, Austria) was used for all data analysis.

A total of 246 consecutive patients (age $48 \pm 15$ years) were included with a mean follow-up of $68 \pm 16$ days after infection (table 1). The majority of patients $(n=224,91 \%)$ did neither require hospital admission nor receive specific therapy $(n=236,96 \%)$. Inpatients were routinely supplemented with oxygen while only two of the 22 hospitalised patients required intensive care unit (ICU) treatment. No comorbidities were reported in 143 patients (58\%) while respiratory $(n=24,10 \%)$ and cardiovascular $(n=22,9 \%)$ were the most frequently reported diseases. Symptoms were present in 244 patients (99\%) at time of infection (range 1-7 symptoms). At follow-up, 113 patients (46\%) remained symptomatic (range 1-3 symptoms). Olfactory loss was present in $\mathrm{n}=162(66 \%)$ at infection and $\mathrm{n}=10(4 \%)$ at follow-up. Corresponding frequencies were: cough 159 (65\%) versus 35 (14\%), pyrexia 145 (59\%) versus $1(<1 \%)$, dyspnoea $76(31 \%)$ versus $79(32 \%)$, sore throat $63(26 \%)$ versus $1(<1 \%)$, rhinitis $61(25 \%)$ versus $2(1 \%)$, thoracic pain 59 (24\%) versus $15(6 \%)$, limb pain $41(17 \%)$ versus $2(1 \%)$, cephalgia $40(16 \%)$ versus $1(<1 \%)$, fatigue 14 $(6 \%)$ versus $3(1 \%)$, diarrhoea $1(<1 \%)$ versus $0(0 \%)$. Significantly more symptoms at time of infection $(3.7 \pm 1.5)$ were reported by patients remaining symptomatic as compared to patients being asymptomatic at follow-up $(3.1 \pm 1.5 ; \mathrm{p}<0.01)$. Symptomatic patients had a significantly lower forced expiratory volume in $1 \mathrm{~s}\left(\mathrm{FEV}_{1}\right)$ of $96 \pm 17 \%$ predicted, vital capacity (VC) of $96 \pm 15 \%$ predicted, and $T_{\mathrm{LCO}}$ of $83 \pm 15 \%$ predicted.

@ERSpublications

About half of the patients recovering from SARS-CoV-2 have persistent symptoms and lower lung function as long as 2 months after infection. This is common even in younger SARS-CoV-2 convalescents with few comorbidities. https://bit.ly/38dyCYB

Cite this article as: Trinkmann F, Müller M, Reif A, et al. Residual symptoms and lower lung function in patients recovering from SARS-CoV-2 infection. Eur Respir J 2021; 57: 2003002 [https://doi.org/10.1183/ 13993003.03002-2020]. 
TABLE 1 Clinical characteristics and lung function

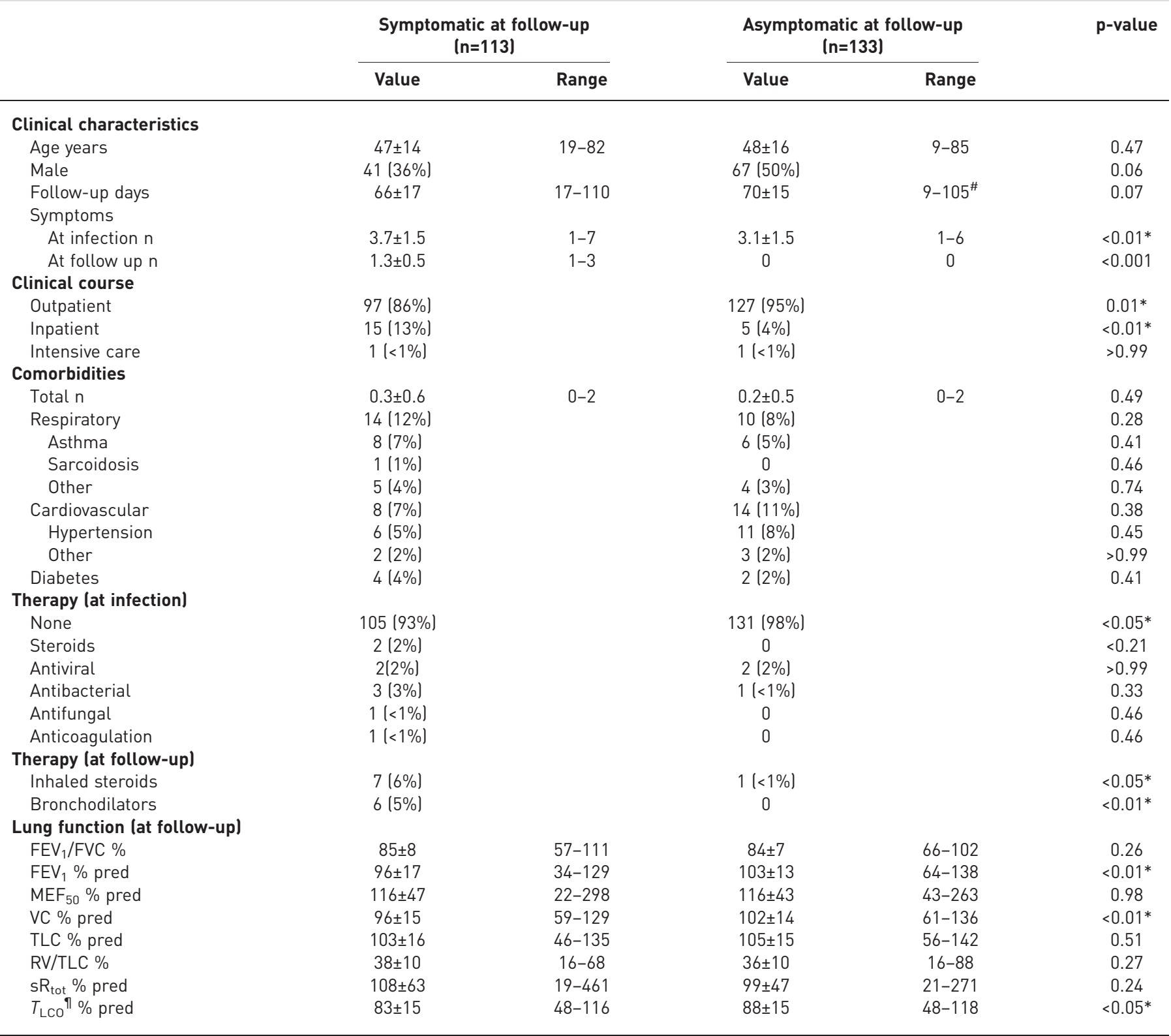

Data are presented as $\mathrm{n}(\%)$ or mean $\pm \mathrm{SD}$, unless otherwise indicated. $F E V_{1}$ : forced expiratory volume in $1 \mathrm{~s}$; (F)VC: (forced) vital capacity; $\mathrm{MEF}_{50}$ : maximum expiratory flow at $50 \%$ of $\mathrm{FVC}$; TLC: total lung capacity; RV: residual volume; sR $\mathrm{tot}_{\text {: }}$ specific total airway resistance; $T_{\mathrm{LCO}}$ : transfer factor of the lung for carbon monoxide. ${ }^{\#}$ : one patient underwent body plethysmography on the day of confirmed infection; ${ }^{\text {: }} T_{\mathrm{LCO}}$ waS available in 159 patients (65\%). *: statistically significant $p<0.05$ (t-test).

$\mathrm{FEV}_{1}, \mathrm{VC}$ and $\mathrm{T}_{\mathrm{LCO}}$ were $103 \pm 13 \%(\mathrm{p}<0.01), 102 \pm 14 \%(\mathrm{p}<0.01)$ and $88 \pm 15 \%(\mathrm{p}<0.05)$ in asymptomatic patients, respectively. CT was available in a subgroup of 17 (7\%). Any abnormality was present in $71 \%$ $(n=12)$ of those patients. The most frequent findings were consolidations $(n=12,71 \%)$, followed by ground glass opacities $(n=11,64 \%)$. Pulmonary embolism and reticulations were detected in $n=2(12 \%)$ each.

We were able to demonstrate that about half of the patients recovering from SARS-CoV-2 have persistent symptoms for more than 2 months after acute infection. Symptomatic patients have significantly reduced lung function, most notably impaired gas transfer, lung volume and central obstruction. To the best of our knowledge, this is the first report to systematically evaluate residual symptoms and lower lung function in convalescents from SARS-CoV-2 infection.

Our findings are especially noticeable as we investigated a cohort with only minor comorbid burden before coronavirus disease 2019 onset. In the initial phase of the current pandemic, high comorbidity and older 
age were identified as predictors of severe cases [3]. However, symptoms and reduced lung function seem to be a relevant burden even in younger populations with only few comorbidities. This is in accordance with recent findings obtained from long-term follow-up of mostly healthy medical staff infected with SARS-CoV-1 during the 2002-2003 pandemic. Initial restriction resolved during the follow-up 15 years later, whereas the incidence of reduced $T_{\mathrm{LCO}}$ and forced expiratory flow at $25-75 \%$ of forced vital capacity $\left(\mathrm{FEF}_{25-75 \%}\right)$ both increased [6]. The latter may correspond to previous findings in seasonal influenza. Small airway obstruction decreased by about $40 \%$ during a 5 -week period after uncomplicated influenza A. Parameters of central obstruction $\left(\mathrm{FEV}_{1}\right)$ and $\mathrm{FEF}_{25-75 \%}$ [7] were within normal, which can be explained by the well-known insensitivity of spirometry. In our data, this may translate to the lower $\mathrm{FEV}_{1}$ (central obstruction) and higher $\mathrm{sR}_{\text {tot }}$ (resistance) in symptomatic patients Therefore, subtle changes in the small airways may be detectable using more sensitive techniques, such as oscillometry or imaging [8]. While CT abnormalities are well described in SARS-CoV-2 [9], little is known about their long-term course. In a subgroup, we found pathologies in an overall of $71 \%$, with consolidations and ground-class opacities being the dominant patterns, which is congruent with the literature [10].

Clinical symptoms at time of presentation are well characterised in both SARS-CoV-1 and SARS-CoV-2. However, little is known about long-term courses. Data from the 2002-2003 pandemic suggest that SARS-CoV-1-induced pulmonary lesions recovered to a greater extent within 1 year after recovery [6]. Reports from Hong Kong and Canada show an increased risk for neuro-psychiatric disorders such as chronic fatigue, depression, pain and sleep disturbances $[11,12]$.

In SARS-CoV-2 infection, the median time from first symptoms to development of dyspnoea is about 58 days $[13,14]$. We could demonstrate a shift of the most frequent symptoms from initially olfactory loss to dyspnoea later on. Dyspnoea then persists over a mean of nearly 70 days in about one third of the patients while olfactory loss was still reported by $4 \%$. We did not find statistically significant or clinically meaningful differences for any of the baseline characteristics in univariate analysis. Female patients showed a trend to being more often symptomatic. Respiratory disorders were numerically slightly more frequent in symptomatic as compared to asymptomatic patients (12\% versus $8 \%)$, with bronchial asthma being the leading entity. In previous reports, asthma was not associated with more severe outcomes in SARS-CoV-2 infection in general $[15,16]$. Although lying within the normal range, we found differences in lung function parameters. The reduction of $T_{\mathrm{LCO}}$ can be potentially attributed to structural damage, as previously seen during the 2002-2003 pandemic. Likewise, reduction of VC might be an expression of musculoskeletal weakness. Long-term consequences remain unknown. Nevertheless, we believe they may already have clinical relevance. First, these changes were associated with clinical symptoms and may even justify medication. Second, the impact of SARS-CoV-2 infection on small airway function may be larger, as previously shown in COPD [17]. This can provide a missing link between lung function and clinical presentation requiring further research. Before the approval of remdesivir in the EU in July 2020, no specific therapy was available for severe SARS-CoV-2 infection [18]. With the majority of our patients not requiring hospital admission, they accordingly did not receive treatment during the initial phase of the pandemic. Patients who did not report symptoms at follow-up were more frequently not treated at time of infection. At follow-up, symptomatic patients received inhaled steroids and bronchodilators more often, potentially indicating more severe disease.

Our study has several strengths, such as the structured clinical work-up and elaborate lung function testing. However, some limitations should also be discussed. Lung function data was not available prior to infection as most patients did not report symptoms requiring diagnostic work-up. No conclusions on health-related quality of life can be drawn and should be investigated in future long-term studies. Selection bias may have been introduced at two points. All patients referred to our hospital participated in the study. However, this may not represent all recovered patients due to the voluntary nature of consulting the medical system. CT was only performed in a small percentage of patients with clinical indication. This may lead to an overrepresentation of symptomatic patients in this subgroup. We do not consider these points as major drawbacks of our study since we intentionally aimed at investigating a rather healthy and young cohort. Severely ill patients are therefore underrepresented in our study, with only $9 \%$ of the hospitalised population requiring ICU treatment. While severely ill patients intuitively have greater impairments at discharge, as recently demonstrated [19], we believe our findings are valuable in this context. The large proportion of outpatient treatment indicates rather mild disease while entailing persistent symptoms in a population that is currently not being considered at risk.

Taken together, symptoms and lower lung function are common even in younger SARS-CoV-2 convalescents with few comorbidities. The long-term effects remain unknown given the most recent occurrence of the disease. Based on extrapolations from the previous SARS pandemic, careful follow-up is warranted in the current situation. Measures should be designed to detect and appropriately manage any persistent or emerging long-term consequences. 
Frederik Trinkmann $\oplus^{1,2}$, Michael Müller ${ }^{1}$, Alexandra Reif ${ }^{1}$, Nicolas Kahn $\oplus^{1}$, Michael Kreuter $^{1}$, Franziska Trudzinski ${ }^{1}$, Monika Eichinger ${ }^{3}$, Claus-Peter Heussel $\oplus^{3,4}$ and Felix J.F. Herth $\oplus^{1}$ for the Lung Network Rhine-Neckar-Region ${ }^{5}$

${ }^{1}$ Pneumology and Critical Care Medicine, Thoraxklinik at University Hospital Heidelberg, Translational Lung Research Center Heidelberg (TLRC), Member of German Center for Lung Research (DZL), Heidelberg, Germany. ${ }^{2}$ Dept of Biomedical Informatics at the Heinrich-Lanz-Center, University Medical Center Mannheim, Heidelberg University, Mannheim, Germany. ${ }^{3}$ Dept of Diagnostic and Interventional Radiology with Nuclear Medicine, Thoraxklinik at University Hospital Heidelberg, Translational Lung Research Center Heidelberg (TLRC), Member of German Center for Lung Research (DZL), Heidelberg, Germany. ${ }^{4}$ Dept of Diagnostic and Interventional Radiology, University Hospital Heidelberg, Heidelberg, Germany. ${ }^{5} \mathrm{~A}$ list of members of the Lung Network Rhine-Neckar-Region can be found in the acknowledgements section.

Correspondence: Felix J.F. Herth, Thoraxklinik at University Hospital Heidelberg, Röntgenstraße 1, 69126 Heidelberg, Germany. E-mail: felix.herth@med.uni-heidelberg.de

Received: 2 Aug 2020 | Accepted: 5 Nov 2020

Clinical trial registration: NCT04456075. Individual patient data will not be made available publicly due to data protection regulations.

Acknowledgements: We thank all patients who agreed to take part in the study, Denise Stock, Julia Kaus and Nicol Breitenfeld for their help in patient management and data handling as well as all members of Lung Network Rhine-Neckar-Region for their support in patient referral.

Lung Network Rhine-Neckar-Region: B. Abenhardt, M. Ast, M. Apprich, E. Brunnemer, C. Corvinus, A. Filusch, R. Freund, T. Greulich, F. Hess-Jähnig, J. Hinrichs-Pavlik, J. Hofbauer, P. Hofbauer, G. Hug, J. Isele, H. Martin, J. Mathews, C. Rehm-Verstege, S. Samardzic, H. Schädler, M. Schulz, U. Steinhauser, P. van Bodegom

Conflict of interest: F. Trinkmann received travel support from Actelion, Berlin Chemie, Boehringer Ingelheim, Chiesi, Novartis, Mundipharma and TEVA as well as speaker or consultation fees from AstraZeneca, Berlin Chemie, Boehringer Ingelheim, Bristol-Myers Squibb, Chiesi, GlaxoSmithKline, Novartis, Roche and Sanofi-Aventis, all outside the submitted work. M. Müller has nothing to disclose. A. Reif has nothing to disclose. N. Kahn received travel support from GSK and Berlin Chemie as well as speaker or consultation fees from Berlin Chemie and Roche, all outside the submitted work. M. Kreuter has nothing to disclose. F. Trudzinski received travel support from Actelion, Berlin Chemie, Chiesi, Novartis, and speaker or consultation fees from Novartis and Berlin Chemie, all outside the submitted work. M. Eichinger reports consultation fees from Roche, outside the submitted work. C-P. Heussel reports speaker or consultation fees from AstraZeneca, Basilea, Bayer, Bracco, Boehringer Ingelheim, Chiesi, Covidien, Essex, Gilead, Grifols, Intermune, Lilly, MEDA Pharma, MSD, Novartis, Roche, Schering-Plough, Siemens, Pfizer and Pierre Fabre, all outside the submitted work. F.J.F Herth has nothing to disclose.

\section{References}

$1 \mathrm{Wu} \mathrm{Z}, \mathrm{McGoogan}$ JM. Characteristics of and important lessons from the coronavirus disease 2019 (covid-19) outbreak in China: summary of a report of 72314 cases from the Chinese Center for Disease Control and Prevention. JAMA 2020; 323: 1239-1242.

2 Oran DP, Topol EJ. Prevalence of asymptomatic SARS-CoV-2 infection: a narrative review. Ann Intern Med 2020; 173: $362-367$.

3 Zhang JJ, Dong X, Cao YY, et al. Clinical characteristics of 140 patients infected with SARS-CoV-2 in Wuhan, China. Allergy 2020; 75: 1730-1741.

4 Cooper BG, Stocks J, Hall GL, et al. The Global Lung Function Initiative (GLI) Network: bringing the world's respiratory reference values together. Breathe (Sheff) 2017; 13: e56-e64.

5 Quanjer PH, Tammeling GJ, Cotes JE, et al. Lung volumes and forced ventilatory flows. Report Working Party Standardization of Lung Function Tests, European Community for Steel and Coal. Official Statement of the European Respiratory Society. Eur Respir J Suppl 1993; 6: Suppl. 16, 5-40.

6 Zhang P, Li J, Liu H, et al. Long-term bone and lung consequences associated with hospital-acquired severe acute respiratory syndrome: a 15-year follow-up from a prospective cohort study. Bone Res 2020; 8: 8 .

7 Hall WJ, Douglas RG, Jr., Hyde RW, et al. Pulmonary mechanics after uncomplicated influenza A infection. Am Rev Respir Dis 1976; 113: 141-148.

8 Trinkmann F, Watz H, Herth FJF. Why do we still cling to spirometry for assessing small airway function? Eur Respir J 2020; 56: 2001071.

9 Zhang R, Ouyang $\mathrm{H}, \mathrm{Fu} \mathrm{L}$, et al. CT features of SARS-CoV-2 pneumonia according to clinical presentation: a retrospective analysis of 120 consecutive patients from Wuhan city. Eur Radiol 2020; 30: 4417-4426.

10 Jin Q, Heussel CP, Kauczor HU, et al. Evolution of CT manifestations in a coronavirus (2019-nCoV) pneumonia patient. Med Klin Intensivmed Notfmed 2020; 115: 412-413.

11 Moldofsky H, Patcai J. Chronic widespread musculoskeletal pain, fatigue, depression and disordered sleep in chronic post-SARS syndrome; a case-controlled study. BMC Neurol 2011; 11: 37.

12 Lam MH, Wing YK, Yu MW, et al. Mental morbidities and chronic fatigue in severe acute respiratory syndrome survivors: long-term follow-up. Arch Intern Med 2009; 169: 2142-2147.

13 Wang $\mathrm{D}, \mathrm{Hu} \mathrm{B}, \mathrm{Hu}$ C, et al. Clinical characteristics of 138 hospitalized patients with 2019 novel coronavirus-infected pneumonia in Wuhan, China. JAMA 2020; 323: 1061-1069.

14 Huang C, Wang Y, Li X, et al. Clinical features of patients infected with 2019 novel coronavirus in Wuhan, China. Lancet 2020; 395: 497-506.

15 Lovinsky-Desir S, Deshpande DR, De A, et al. Asthma among hospitalized patients with COVID-19 and related outcomes. J Allergy Clin Immunol 2020; 146: 1027-1034.e4. 
16 Beurnier A, Jutant EM, Jevnikar M, et al. Characteristics and outcomes of asthmatic patients with COVID-19 pneumonia who require hospitalisation. Eur Respir J 2020; 56: 2001875.

17 Crisafulli E, Pisi R, Aiello M, et al. Prevalence of small-airway dysfunction among COPD patients with different GOLD stages and its role in the impact of disease. Respiration 2017; 93: 32-41.

18 Beigel JH, Tomashek KM, Dodd LE, et al. Remdesivir for the treatment of Covid-19 - preliminary report. $N$ Engl J Med 2020; 383: 994.

19 Mo X, Jian W, Su Z, et al. Abnormal pulmonary function in COVID-19 patients at time of hospital discharge. Eur Respir J 2020; 55: 2001217.

Copyright @ERS 2021

This version is distributed under the terms of the Creative Commons Attribution Non-Commercial Licence 4.0. 